\title{
Care of Immunocompromised Patients with Head and Neck Cancer During the COVID-19 Pandemic: Two Challenging and Informative Clinical Cases
}

\author{
Alyssa M. Civantos et al. ${ }^{1}$ \\ ${ }^{1}$ Affiliation not available
}

April 28, 2020

\begin{abstract}
Background and Methods: There is an added level of complexity in the management of head and neck cancer patients with underlying immunosuppressive disorders during the COVID-19 pandemic. Head and neck oncologists are tasked with balancing the dual risks of cancer progression in the setting of impaired tumor immunity and increased susceptibility to life-threatening complications from exposure to viral infection for patients and providers. Through two cases of immunocompromised patients with newly diagnosed head and neck malignancies, we aim to provide guidance to clinicians struggling with how to best counsel and manage this unique subset of patients under these difficult circumstances. Results: After careful consideration of the options, we took different approaches in the care of these two patients. Conclusions: Ultimately, there is no uniform set of rules to apply to this heterogeneous group of immune-compromised patients. We provide some general principles to help guide patient management during the current pandemic.
\end{abstract}

\section{Full author list}

Alyssa M. Civantos, BA, ${ }^{1}$ Ryan M. Carey, MD ${ }^{2}$ Gary R. Lichtenstein, MD, ${ }^{3}$ John N. Lukens, MD ${ }^{4}$ Roger B. Cohen, $\mathrm{MD},{ }^{5}$ Christopher H. Rassekh, $\mathrm{MD}^{2}$

${ }^{1}$ Perelman School of Medicine at the University of Pennsylvania, Philadelphia, PA

${ }^{2}$ Department of Otorhinolaryngology: Head and Neck Surgery, University of Pennsylvania, Philadelphia, PA

${ }^{3}$ Department of Medicine, Division of Gastroenterology, University of Pennsylvania, Philadelphia, PA

${ }^{4}$ Department of Radiation Oncology, University of Pennsylvania, Philadelphia, PA

${ }^{5}$ Department of Medical Oncology, University of Pennsylvania, Philadelphia, PA

\section{Introduction}

We currently find ourselves in unprecedented times. The measures needed to control the COVID-19 outbreak are directly at odds with providing comprehensive cancer care. Across the world, people are being told to stay home and severely limit their activities. But the treatment of head and neck cancer requires multidisciplinary collaboration, which directly translates to significant exposure and interactions with many different people in hospital systems. Early data from the epidemic in China suggest that a cancer diagnosis is a risk factor for severe events from COVID-19, especially if the patient recently underwent chemotherapy or surgery. ${ }^{1-4}$ Patients with cancer and iatrogenic immune suppression for the treatment of other medical conditions are likely at even greater risk of complications and death from viral infection. 
Malignant tumors of the head and neck account for approximately $3 \%$ of all cancers in the United States, with about 53,260 Americans expected to be diagnosed in 2020.5 Prompt diagnosis and treatment are critical for increasing survival and preserving organ function and quality of life. A systematic review on time to diagnosis or treatment in oral, pharyngeal, and laryngeal cancer patients found higher stage and inferior survival with longer treatment delays. ${ }^{6,7}$

During the current pandemic, any decision that could lead to airway emergencies or more extensive surgeries in the near future has the potential for putting health care providers at greater risk of COVID-19 exposure. ${ }^{8-10}$ Aerosol-generating procedures, including tracheal intubation, tracheotomy, non-invasive ventilation, and manual ventilation, are associated with increased risks of acute respiratory infections among health care workers. ${ }^{11}$ Anecdotal reports from Wuhan, China, report higher rates of infection specifically among otolaryngologists. ${ }^{12-15}$ Personal protective equipment (PPE) is a currently scarce but essential resource for the aerosolizing procedures performed by otolaryngologists.

The decision to perform surgery for mucosal cancer is currently difficult in all head and neck cancer patients, but particularly complicated in patients with a secondary diagnosis or medical therapy leading to an immunocompromised state, as their risk of infection with SARS-CoV-2 is surely even more pronounced. A diagnosis of cancer in an already immunocompromised patient is also a time-sensitive matter, as impaired immunity can increase the growth and spread of a cancer. ${ }^{16,17}$ Immunocompromised patients with COVID19 are also thought to be more likely to have severe adverse events, although the data are still limited.1,18 Combining the two factors of a cancer diagnosis and an immune suppressed state intensifies the gravity and complexity of the situation. We report two presentations of head and neck cancer in immunocompromised patients in the setting of this global pandemic and discuss our clinical rationale for the different approaches taken in each case.

Case Reports:

\section{Case 1:}

A 46-year-old male with a history of aplastic anemia and allogeneic hematopoietic stem cell transplantation complicated by chronic graft versus host disease (GVHD) resulting in multiple past squamous cell carcinomas (SCC) of the oral cavity presented with a new \souttongue lesion. In the past he has undergone multiple resections of tongue and buccal mucosa cancers with reconstructions, a left neck dissection, and adjuvant radiotherapy. His course has been complicated by a difficult airway, vocal cord paralysis, an occluded left carotid stent, and a complex neck abscess requiring long-term antibiotic prophylaxis.

A new oral tongue lesion was first identified on routine follow-up in January 2020. The patient was asymptomatic without pain, bleeding, or difficulty eating. Exam demonstrated a lesion of the right lateral tongue with thickened leukoplakia and central depression. There was mild erythema of the mucosa but no erythroplakia. The left lateral tongue had minimal leukoplakia similar to prior exams. There were no palpable cervical lymph nodes or visible lesions on flexible nasopharyngolaryngoscopy. On repeat exam a few weeks later, the patient reported new tongue tenderness, worse on the right side. The physical appearance of the lesion was unchanged other than mildly increased thickness. Given worsening symptoms, the decision was made to perform an in-office biopsy.

Biopsy of the tongue lesion revealed squamous cell carcinoma, at least in situ, and hyperkeratotic squamous mucosa with lichenoid chronic inflammation. Pathology noted that invasive cancer could not be excluded based on the biopsy specimen. We therefore planned a transoral partial glossectomy for excision of the new tongue lesion with intraoperative assessment of margins and possible split thickness skin graft.

At this point, COVID-19 had entered our institution. Although surgery was the ideal approach to the diagnosis and treatment, we decided to delay intervention for the time being given his significant immunocompromised state and the small, relatively indolent nature of a lesion that could be monitored visually with relative ease. He is currently home quarantined with care coordinated via telemedicine including weekly photographic updates of the tongue lesion (Fig. 1). 


\section{Case 2:}

A 39-year-old female, never-smoker, with a history of squamous cell carcinoma in situ of the right true vocal cord, longstanding Crohn's disease, and gastroesophageal reflux presented with a cough and sore throat. The prior right vocal cord lesion had been treated with microlaryngoscopy and KTP laser excision by an outside otolaryngologist seven months earlier. The pathology was consistent with SCC in situ with negative margins. Her Crohn's disease has been difficult to control with oral involvement and prior requirement for multiple immunosuppressants and steroids, including most recently the systemic biologic ustekinumab (Stelara ${ }^{\circledR}$ ). Gastroenterology planned to switch the patient to vedolizumab (Entyvio ${ }^{\circledR}$ ) in place of ustekinumab given ongoing uncontrolled GI symptoms and the presence of the head and neck neoplasm. Vedolizumab is a more gastrointestinal-selective agent, with less systemic immunosuppression.

At presentation she reported a number of laryngeal symptoms including constant cough, hoarseness, and sore throat, without shortness of breath. On nasopharyngolaryngoscopy, a mass of the right anterior/mid true cord was observed. The entire vocal fold was noted to be edematous and erythematous, but vocal cord mobility was normal. There were no palpable cervical lymph nodes. The patient was counseled on the need for tissue biopsy of the concerning vocal cord lesion.

Microdirect laryngoscopy with biopsy of the right vocal cord mass was performed in late January 2020. The lesion involved the right anterior vocal cord without involvement of the anterior commissure, very limited involvement of the inferior aspect of the vocal cord and suspicion for possible early infraglottic extension. The lesion was noted to be partly submucosal, very firm, and adherent to the underlying muscle/ligament (Fig. 2). Pathology was positive for invasive, well-differentiated, keratinizing SCC. She was therefore diagnosed with a stage I (cT1aN0M0) SCC of the right vocal fold.

At this point, COVID-19 had entered our institution. Standard of care options would normally include open or endoscopic cordectomy or definitive radiotherapy. Radiation Oncology favored primary surgical resection over radiotherapy given the risk of increased radiation toxicity in a patient with active Crohn's disease with oral cavity involvement, the risk of exposure to SARS-CoV-2 from other patients and healthcare providers during a typical 28-fraction (six-week) course of radiotherapy, which would have corresponded to the predicted peak surge in COVID-19 cases in our region, and her young age and hypothetical risk of secondary malignancy. After discussion of the risks and benefits of the different options with the patient and in collaboration with our multidisciplinary team, she decided to undergo laser cordectomy.

In order to minimize aerosolization, the procedure was done with sharp (cold) technique instead of laser. It was felt that an outpatient procedure was preferable with avoidance of open partial laryngectomy with imbrication and tracheostomy. At the time of surgery, the tumor was considerably more extensive in terms of infraglottic spread than had been anticipated and had likely progressed over a very short interval since the original diagnosis. She was discharged after surgery and instructed to take oral hydroxychloroquine (Plaquenil) $400 \mathrm{mg}$ twice daily on day one, then $200 \mathrm{mg}$ twice daily for four days per an Infectious Disease consultant. Final pathology revealed a keratinizing, well differentiated, invasive squamous cell carcinoma of the right vocal cord with negative but close margins, and was pathologically staged as T2a. Tumor board consensus was to follow her by telemedicine for the next several weeks and have her return for an in-person visit after the anticipated viral infection "surge" in our community, and if she were to recur, consideration of radiotherapy or supracricoid partial laryngectomy. At this time, the patient is recovering well post-operatively, with her care being coordinated via telemedicine.

\section{Discussion}

In this report we present two cases of immunocompromised patients presenting with head and neck cancer with the goal of using their management as illustrations that exemplify how therapeutic decisions might be made in this extremely vulnerable population at a unique time in the practice of medicine.

In Case 1, a patient developed another early oral tongue lesion on a background of a very complicated history of prior oral cavity cancers and chronic GVHD after allogeneic stem cell transplant for aplastic anemia. In 
this case, we determined that the risk of potential COVID-19 exposure outweighed the risk of delaying his treatment for a few months. A number of factors contributed to our decision. The location and size of the lesion on the tongue was thought to not put the patient at risk for airway obstruction. The lesion was easy visualized by the patient and clinician through telemedicine visits, and the patient felt comfortable sharing photographs regularly. Finally, the anticipated delay in definitive therapy of a few months was thought unlikely to significantly alter his functional outcome and overall survival.

In Case 2, we presented a recurrent early stage glottic lesion in a patient on chronic immunosuppressive medications for Crohn's disease. The biologic agent used to treat her inflammatory bowel disease was modified to use a less systemically immunosuppressive agent. The benefits of proceeding with surgery immediately were felt to outweigh the risk of possible COVID-19 exposure. The main reasons for this decision were the implications of rapid cancer progression on her overall outcome, the potential airway risk, and inability to monitor with telemedicine. The decision to recommend surgery over definitive radiotherapy was multifactorial but incorporated the heightened risk of exposure to SARS-CoV-2 during a typical six-week course of radiotherapy, which in her case would have corresponded to the predicted peak surge in COVID-19 cases in our region.

During this pandemic, especially in situations where the benefits of each modality are comparable, we should consider the degree of immunosuppression caused by our therapies as well as the risks of viral exposure to the immunocompromised patient. There is a period of immunosuppression in the immediate post-operative period due to a variety of metabolic and endocrine responses with studies showing increases in glucocorticoids and decreases in the number and function of critical leukocyte subpopulations for up to 30 days; the extent of the change is related to the magnitude of surgical insult. ${ }^{19}$ Additional studies also have shown that the immunosuppressive effects of surgery can be minimized by adequate pain control and the use of minimally invasive surgical techniques.

While surgery may put the patient at acute risk for exposure to COVID-19, chemotherapy may result in further immunosuppression, and radiotherapy typically requires daily visits to an outpatient clinic over a period of five to seven weeks. ${ }^{20-25}$ The latter will increase the potential exposure to SARS-CoV-2. The overall risk to the immunocompromised patient of acquiring a COVID-19 infection is probably greater with radiation therapy with or without chemotherapy than after a single visit to the hospital for surgery, even if that one visit requires tracheostomy and hospitalization. However, sufficient data are not available to reach definitive conclusions about these competing risks at this time.

A discussion with an Infectious Disease specialist about the potential benefit of medications such as hydroxychloroquine, a drug with some preliminary, but limited, evidence of inhibition of SARS-CoV-2 in vitro and viral load reduction in COVID-19 patients, may be warranted for the post-operative period, as was agreed upon in Case 2. ${ }^{26-29}$ The importance of home isolation should be stressed with all of these patients.

The current rule of thumb in the face of this pandemic is cancellation of elective appointments and surgeries; however, no cancer treatment is truly elective. ${ }^{30-32}$ Decision-making for all patients with operable cancers requires a nuanced approach, especially when there is the added complication of co-morbid immunosuppression as in the two cases we present. We favor operating when there is a concern for future airway obstruction or life-threatening bleeding if treatment is delayed. If the decision is made to operate, appropriate PPE, such as an N95 mask with a face shield and eye protection, is of utmost importance to protect the entire surgical team as well as the patient. ${ }^{8,13-15}$ Telemedicine appointments and photographic monitoring should be used whenever feasible in order to allow home isolation. Most importantly, we recommend an honest discussion of the situation with the patient, taking into account their comfort and goals of care.

\section{Conclusion}

The COVID-19 pandemic continues to present innumerable unique challenges throughout the healthcare system. Otolaryngology is an especially challenging subspecialty at this time, with the intrinsic exposure risks to providers of procedures that generate aerosols. Practice is even more complicated when patients present with complex cancers and coincident immunosuppression. Clinical decision-making requires careful 
weighing of the competing risks of various approaches, and honest communication with the patient. A comprehensive, multidisciplinary approach, including the addition of specialties such as Infectious Disease, is the preferred approach to managing these patients in these unusual circumstances.

References

1. Zhang L, Zhu F, Xie L, et al. Clinical characteristics of COVID-19-infected cancer patients: A retrospective case study in three hospitals within Wuhan, China. Annals of Oncology . 2020. doi: https://doi.org/10.1016/j.annonc.2020.03.2

2. Liang W, Guan W, Chen R, et al. Cancer patients in SARS-CoV-2 infection: a nationwide analysis in China. The Lancet Oncology . 2020;21 (3):335-37. doi: https://doi.org/10.1016/S1470-2045(20)30096-6

3. Wu Z, McGoogan JM. Characteristics of and Important Lessons From the Coronavirus Disease 2019 (COVID-19) Outbreak in China. JAMA . Published online February 24, 2020. doi: 10.1001/jama.2020.2648

4. Yong-Cong C, Wang W, Li C, et al. Treating head and neck tumors during the SARS-CoV-2 epidemic, 2019-2020: Sichuan Cancer Hospital. Authorea . April 01, 2020. doi: https://doi.org/10.22541/au.158576817.72109459

5. Siegel RL, Miller KD, Jemal A. Cancer statistics, 2020. CA: A Cancer Journal for Clinicians . 2020;70 :7-30. doi: https://doi.org/10.3322/caac.21590

6. Schutte HW, Heutink F, Wellenstein DJ, et al. Impact of Time to Diagnosis and Treatment in Head and Neck Cancer: A Systematic Review. Otolaryngology-Head and Neck Surgery . 2020. doi: https://doi.org/10.1177\%2F019459982

7. Murphy CT, Galloway TJ, Handorf EA, et al. Survival Impact of Increasing Time to Treatment Initiation for Patients With Head and Neck Cancer in the United States. Journal of Clinical Oncology . 2016;34 (2):169-78. doi: https://dx.doi.org/10.1200\%2FJCO.2015.61.5906

8. Givi B, Schiff BA, Chinn SB, et al. Safety Recommendations for Evaluation and Surgery of the Head and Neck During the COVID-19 Pandemic. JAMA Otolaryngology-Head \& Neck Surgery . Published online March 31, 2020. doi: doi:10.1001/jamaoto.2020.0780

9. Vargas M, Servillo G. Improving staff safety during tracheostomy in COVID-19 patients. Authorea . March 31, 2020. doi: https://doi.org/10.22541/au.158567936.68111075

10. Rassekh CH, Jenks CM, Ochroch EA, Douglas JE, O'Malley Jr. BW, Weinstein GS. Management of the Difficult Airway in the COVID-19 Pandemic: An Illustrative Complex Head and Neck Case Scenario. Authorea . April 02, 2020. doi: https://doi.org/10.22541/au.158585426.67421372

11. Tran K, Cimon K, Severn M, Pessoa-Silva CL, Conly J. Aerosol Generating Procedures and Risk of Transmission of Acute Respiratory Infections to Healthcare Workers: A Systematic Review. PLoS ONE . 2012;7 (4):e35797. doi: https://dx.doi.org/10.1371\%2Fjournal.pone.0035797

12. Europe's Doctors Repeat Errors Made in Wuhan, China Medics Say. Bloomberg News. Published March 17, 2020. Accessed April 5, 2020. https://www.bloomberg.com/news/articles/2020-03-17/europe-s-doctorsgetting-sick-like-in-wuhan-chinese-doctors-say

13. Workman AD, Welling DB, Carter BS, et al. Endonasal instrumentation and aerosolization risk in the era of COVID-19: simulation, literature review, and proposed mitigation strategies. International Forum of Allergy \&3 Rhinology . 2020. doi: https://doi.org/10.1002/alr.22577

14. Kowalski LP. COVID-19 pandemic: effects and evidence-based recommendations for otolaryngology and head and neck surgery practice. Authorea . March 31, 2020. doi: https://doi.org/10.22541/au.158567937.74092406

15. Bann DV, Patel VA, Saadi R, et al. Impact of Coronavirus (COVID-19) on Otolaryngologic Surgery: A Brief Commentary. Authorea. March 31, 2020. doi: https://doi.org/10.22541/au.158567936.61156499

16. Schreiber RD, Old LJ, Smyth MJ. Cancer Immunoediting: Integrating Immunity's Roles in Cancer Suppression and Promotion. Science . 2011;331 (6024):1565-70. doi: 10.1126/science.1203486 
17. Schoenfeld JD. Immunity in Head and Neck Cancer. Cancer Immunology Research . 2015;3 (1):12-17. doi: https://dx.doi.org/10.1158/2326-6066.CIR-14-0205

18. Preliminary Estimates of the Prevalence of Selected Underlying Health Conditions Among Patients with Coronavirus Disease 2019 - United States, February 12-March 28, 2020. MMWR Morb Mortal Wkly Rep . 2020;69 :382-86. doi: http://dx.doi.org/10.15585/mmwr.mm6913e2

19. Hogan BV, Peter MB, Shenoy HG, Horgan K, Hughes TA. Surgery induced immunosuppression. The Surgeon . 2011;9 (1):38-43. doi: https://doi.org/10.1016/j.surge.2010.07.011

20. Carvalho HdA, Villar RC. Radiotherapy and immune response: the systemic effects of a local treatment. Clinics (Sao Paulo) . 2018;73 (suppl 1):e557s. doi: 10.6061/clinics/2018/e557s

21. Verastegui EL, Morales RB, Barrera-Franco JL, Poitevin AC, Hadden J. Long-term immune dysfunction after radiotherapy to the head and neck area. Int Immunopharmacol . 2003;3 (8):1093-104. doi: https://doi.org/10.1016/S1567-5769(03)00013-4

22. Vermorken JB, Remenar E, Van Herpen C, et al. Cisplatin, Fluorouracil, and Docetaxel in Unresectable Head and Neck Cancer.New England Journal of Medicine . 2007;357 (17):1695-704. doi: 10.1056/NEJMoa071028

23. Posner MR, Hershock DM, Blajman CR, et al. Cisplatin and Fluorouracil Alone or with Docetaxel in Head and Neck Cancer. New England Journal of Medicine . 2007;357 (17):1705-15. doi: DOI: 10.1056/NEJMoa070956

24. Izawa N, Onozawa Y, Hikosaka T, et al. Efficacy and feasibility of docetaxel, cisplatin, and 5-fluorouracil induction chemotherapy for locally advanced head and neck squamous cell carcinoma classified as clinical nodal stage N2c, N3, or N2b with supraclavicular lymph node metastases. Int J Clin Oncol . 2015;20 (3):455-62. doi: https://doi.org/10.1007/s10147-014-0749-4

25. Rasmussen L, Arvin A. Chemotherapy-induced immunosuppression.Environmental Health Perspectives . 1982;43 :21-25. doi: https://doi.org/10.1289/ehp.824321

26. Colson P, Rolain J-M, Lagier J-C, Brouqui P, Raoult D. Chloroquine and hydroxychloroquine as available weapons to fight COVID-19. Int J Antimicrob Agents . 2020:105932. doi: https://doi.org/10.1016/j.ijantimicag.2020.105932

27. Gautret P, Lagier J-C, Parola P, et al. Hydroxychloroquine and azithromycin as a treatment of COVID19: results of an open-label non-randomized clinical trial. Int J Antimicrob Agents . 2020:105949. doi: https://doi.org/10.1016/j.ijantimicag.2020.105949

28. Wang M, Cao R, Zhang L, et al. Remdesivir and chloroquine effectively inhibit the recently emerged novel coronavirus (2019-nCoV) in vitro. Cell Res . 2020;30 (3):269-71. doi: https://dx.doi.org/10.1038\%2Fs41422020-0282-0

29. Information for Clinicians on Therapeutic Options for COVID-19 Patients. CDC: Centers for Disease Control and Prevention. Updated March 21, 2020. Accessed April 5, 2020. https://www.cdc.gov/coronavirus/2019ncov/hcp/therapeutic-options.html

30. New Recommendations Regarding Urgent and Nonurgent Patient Care. American Academy of OtolaryngologyHead and Neck Surgery. Accessed April 3, 2020. https://www.entnet.org/content/new-recommendationsregarding-urgent-and-nonurgent-patient-care

31. Ueda M, Martins R, Hendrie P, et al. Managing Cancer Care During the COVID-19 Pandemic: Agility and Collaboration Toward a Common Goal.J Natl Compr Canc Netw .18 (4):1-4. doi: 10.6004/jnccn.2020.7560

32. Siddiqui S. CMS adult elective surgery and procedures recommendations: limit all non-essential planned surgeries and procedures, including dental, until further notice. Published Mar 18, 2020. Accessed April 3, 2020. https://www.cms.gov/files/document/31820-cms-adult-elective-surgery-and-procedures-recommendations.pdf 
Figures

\section{Hosted file}

Figure_1.tiff available at https://authorea.com/users/5588/articles/440472-care-of-immunocompromisedpatients-with-head-and-neck-cancer-during-the-covid-19-pandemic-two-challenging-and-informativeclinical-cases

Figure 1. Photograph of patient 1's tongue lesion uploaded to the secure electronic healthcare portal for review by the head and neck surgeon.

\section{Hosted file}

Figure_2.tiff available at https://authorea.com/users/5588/articles/440472-care-of-immunocompromisedpatients-with-head-and-neck-cancer-during-the-covid-19-pandemic-two-challenging-and-informativeclinical-cases

Figure 2. Photograph of patient 2's right vocal cord squamous cell carcinoma. 